\title{
Low Level Light Therapy as an Adjunct Treatment for Meibomian Gland Dysfunction
}

\author{
Karl Stonecipher ${ }^{1 *}$, Casey Komm ${ }^{1}$ and Richard Potvin ${ }^{2}$ \\ ${ }^{1}$ Physicians Protocol Greensboro, NC, USA \\ ${ }^{2}$ Science in Vision, Bend, OR, USA \\ *Corresponding Author: Karl Stonecipher, Physicians Protocol Greensboro, NC, \\ USA.
}

Received: September 20, 2020

Published: October 07, 2020

(C) All rights are reserved by Karl

Stonecipher., et al.

\begin{abstract}
Importance: This study highlights the adjunctive value of low-level light therapy (LLLT) in the management of meibomian gland dysfunction (MGD).

Background: To evaluate the effects of LLLT treatment on clinical measures of dry eye related to MGD in patients unresponsive to previous therapies.

Design: A retrospective chart review of patients treated at one site.

Participants: Fifty eyes of 25 treated patients who were documented treatment failures with previous pharmaceuticals and/or devices were evaluated.

Methods: LLLT treatment consisted of 3 treatments in one week, with adjunct use of a topical steroid/antibiotic combination. The post-treatment diagnostic measures had to have been made 3 to 5 weeks after the last LLLT treatment.

Main Outcome Measures: Results included changes in the graded MGD score (grading scale 0 - 4), tear breakup time (TBUT), an Ocular Surface Disease Index (OSDI) questionnaire score and lissamine green (LG) staining.

Results: Significant improvements in the mean OSDI score ( $p=0.002)$, MGD grading $(p<0.001)$, TBUT ( $<<0.001)$ and both nasal and temporal LG staining ( $<$ 0.02) were observed after treatment. An MGD grade reduction of 1 or more was observed in $72 \%$ of eyes (36/50), and 2 or more in $32 \%$ of eyes (16/50). There were no ocular or facial adverse events or side effects related to the treatment. Conclusion: The application of LLLT over a short series of sessions for the treatment of MGD appeared to be beneficial in the majority of patients who had failed to improve with alternative pharmaceutical and device interventions.

Keywords: LLLT; Low Level Light Therapy; Meibomian Gland Dysfunction; Ocular Surface Disease Index (OSDI); Dry Eye Disease (DED)
\end{abstract}

\section{Introduction}

Dry eye disease (DED) is a condition that affects hundreds of millions of people worldwide. ${ }^{1}$ The key characteristic of DED is tear film instability. Poor tear coverage of the cornea produces inflammation, which if left unmanaged can ultimately cause damage to the ocular surface [1]. Poor tear coverage can be the result of an insufficient production of tears (aqueous deficiency) or excessive evaporation of tears (evaporative dry eye, or EDE); most DED involves a combination of the two. Increased tear osmolarity due to EDE causes an inflammatory cascade as the eye responds, further destabilizing the tear film [2].

Meibomian gland dysfunction (MGD) is recognized as a leading cause of DED [3-5]. Gland dropout due to age and poor quality meibum are two well-established contributing factors. The gland ducts become clogged, initially with overly viscous meibum and 
in chronic conditions as a result of keratinized cells on the duct margin. The traditional treatment of MGD has ranged from warm compresses and lid scrubs to topical or systemic pharmaceutical therapy, but in recent years new devices to warm the lids and/or to manually express viscous meibum have been developed [6-8].

A less invasive non-pharmaceutical alternative to manually manipulating the lids (a process which can be difficult with some eyes and uncomfortable for some patients) involves the application of low levels of red light to the upper face - this is termed low level light therapy (LLLT). Light emitting diodes (LEDs) of a specific wavelength can cause photon interference, resulting in the penetration of therapeutic levels of light below the skin. LLLT of the correct wavelength can initiate a photochemical cascade (photobiomodulation) which produces non-thermal and non-traumatic cellular photoactivation, reported to result in repair to damaged or compromised cells and improved cellular function in normal cells [9]. The ability of LLLT to activate fibroblasts and enhance collagen synthesis is the basis for the efficacy of skin rejuvenation treatments [10]. The successful application of LLLT for dermatological use has been documented $[11,12]$ and the mechanism of action indicates success in ophthalmic applications is likely $[9,13]$. In early studies of ophthalmic applications the effects of LLLT appear promising $[14,15]$. Potential contributing effects include the photoactivation above, as well as the warming effect on meibum and minimizing demodex mite infestation through increase phagocyte activity [16].

A relatively new device capable of delivering LLLT treatment is the EPI-C device (Espansione Marketing S.p.A., Bologna, Italy), using only the LLLT technology. The device is $510(\mathrm{~K})$ approved in the USA and CE-Marked in Europe under either the EYE-LIGHT or MEIBOMASK brands. The mask contains a matrix of LEDs (wavelength $633 \pm 10 \mathrm{~nm}$, with an emission power of $100 \mathrm{~mW} / \mathrm{cm}^{2}$ ). LLLT treatments can be provided to patients in-office, with no lid manipulation required and no gel. The mask fits over the face, covering the forehead and periorbital area and delivers energy directly over the upper and lower lids. Figure 1 shows one author (KS) with the mask activated for treatment. Treatments are typically 15 minutes long; in that time, the total fluence in the treated area is 110 joules/ $\mathrm{cm}^{2}$. Video thermography has demonstrated the effectiveness of the mask in endogenously heating the periorbital area, a necessary condition for treatment to be successful [16].

The current study was a retrospective chart review conducted to evaluate the effects of LLLT treatment on the signs and symp- toms of dry eye, evaluated through a subjective questionnaire, tear film breakup time, MGD severity grade as determined using a subjective scale and lissamine green staining.

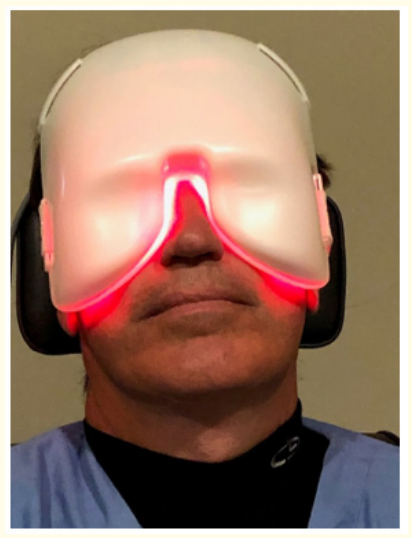

Figure 1

\section{Patients and Methods}

This was a retrospective chart review of patients treated for MGD at one site using LLLT, after recorded failure of previous treatment with pharmaceuticals (oral and/or topical) or other devices, including i-LUX (Alcon, Fort Worth, TX, USA) and Lipiflow (Johnson and Johnson Vision, Jacksonville, FL, USA). The study was approved by an institutional review board (Salus IRB, Austin, TX), which also granted a waiver of informed consent based on the deidentification of the patients' clinical data.

All patients were treated with the EPI-C LLLT device, using the LLLT Mask only. A topical combination antibiotic/steroid combination (gatifloxacin/prednisolone acetate) was also prescribed for use three times per day in both eyes. Standard treatment at the site involved 15 minutes of LLLT applied three times in the course of a week, with 24 to 48 hours between treatments. The use of the topical combination medication continued after the LLLT treatment was completed. Signs and symptoms of DED were evaluated before treatment and 3 - 5 weeks after the third treatment. Any adverse responses to therapy were also documented.

Patients with allergies to any of the proposed treatments, or who were diagnosed with conditions which would preclude their exposure to low-level light therapy were not treated. Patients treated with additional concurrent dry eye therapies were excluded from the data collection, as were pregnant or lactating females and any patient participating in any clinical study at the time of their treatment. 
The clinical evaluation of patients involved several standard measures. Patient scores from the Ocular Surface Disease Index (OSDI) questionnaire were used to assess subjective symptoms related to dry eye [17]. In addition, an MGD grading scale was used to indicate the quality and quantity of meibum that could be expressed; this scale has been described in a previous publication [18]. The TBUT (in seconds) was also recorded, measured subjectively by the clinician at the slit lamp. Lissamine green (LG) staining in the nasal, temporal and central regions of eye was also evaluated on the Oxford grading scale [19]. Endpoints of interest included changes in the OSDI, TBUT, MGD grade and LG staining from pretreatment to post treatment. All patients were monitored for potential side effects related to the LLLT treatment.

Relevant clinical data from files eligible for study inclusion were extracted until 25 records had been assembled. Data were collated and statistical analyses were performed using the Statistical data analysis software system, version 12 (TIBCO Software Inc., Palo Alto, CA, USA). A repeated-measures analysis of variance (ANOVA) was used to compare OSDI and TBUT scores, while the Wilcoxon matched-pairs test was used to evaluate the categorical measures before and after treatment. A p-value $\leq 0.05$ was considered statistically significant.

\section{Results}

A total of 25 patients were successfully identified as eligible to include in the analysis. Nineteen of them were female. The average age was $64 \pm 10$ years, ranging from 45 to 82 years. The treatments of all patients were initiated in January and February 2020. The average time from the final LLLT treatment to the final diagnostic evaluation was $25 \pm 5$ days, ranging from 21 to 36 days.

A histogram of the OSDI scores before and after treatment is shown in figure 2 . The mean score was significantly lower after treatment ( $36.9 \pm 22.2$ before to $29.4 \pm 21.2$ after, $p=0.002$ ). Overall, $80 \%$ of patients $(20 / 25)$ had a decrease in their OSDI score, with $36 \%$ of patients $(9 / 25)$ showing a decrease of 10 or more points.

Figure 3 shows the distribution of TBUT before and after LLLT treatment. The mean breakup time was statistically significantly higher after treatment, increasing from $7.2 \pm 2.2$ to $8.6 \pm 2.0$ seconds $(\mathrm{p}<0.001)$.

Table 1 contains the scoring for the MGD and LG grading, with MGD on a 0 - 4 scale and LG on a 0 - 5 scale. The analyses following relate to these numbers. Both pre-treatment and post-treatment scores are shown. Lissamine green staining was evaluated temporally, centrally and nasally.

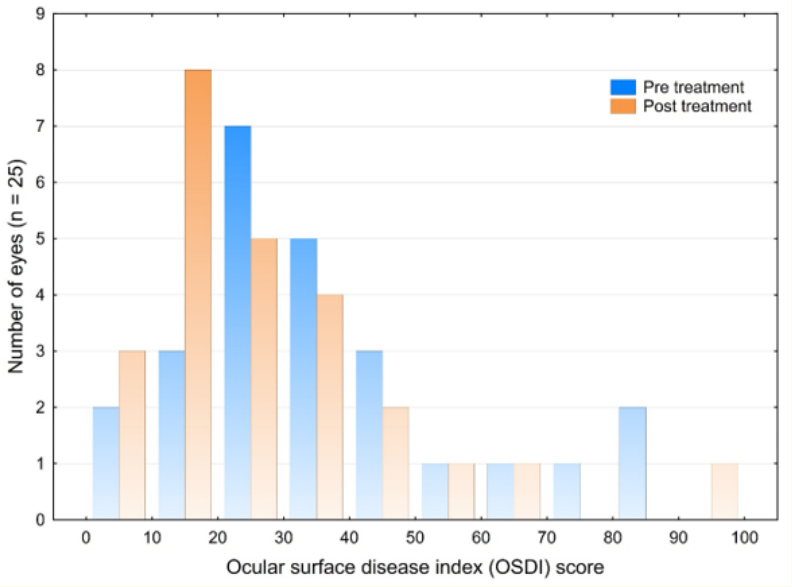

Figure 2: The distribution of ocular surface disease index scores pre and post treatment.

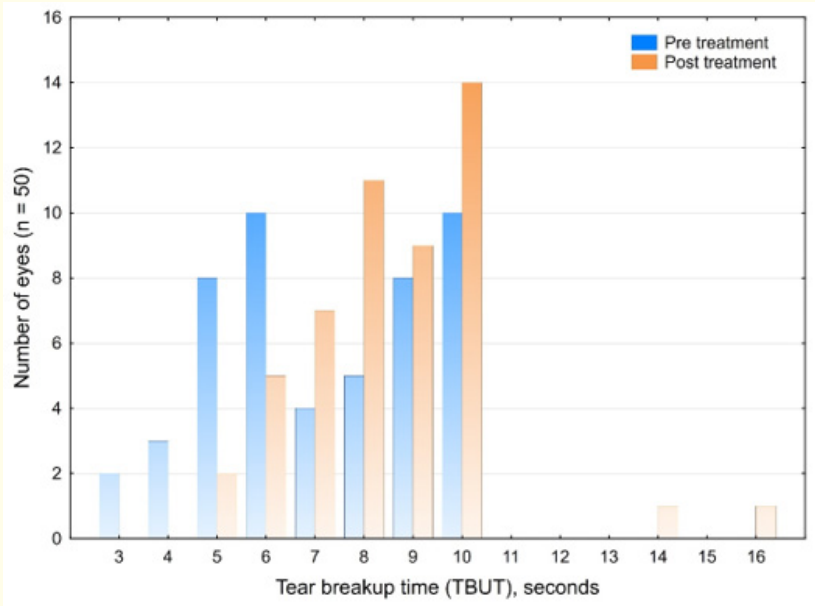

Figure 3: The distribution of tear breakup time before and after treatment.

\begin{tabular}{|l|c|c|c|c|c|c|c|}
\hline & & \multicolumn{7}{|c|}{ Grade } \\
\hline \multirow{2}{*}{ MGD grading } & & $\mathbf{0}$ & $\mathbf{1}$ & $\mathbf{2}$ & $\mathbf{3}$ & $\mathbf{4}$ & $\mathbf{5}$ \\
\cline { 2 - 8 } & Pre & & 6 & 17 & 15 & 12 & n/a \\
\cline { 2 - 8 } & Post & 5 & 20 & 16 & 8 & 1 & n/a \\
\hline \multirow{2}{*}{ LG - nasal } & Pre & 9 & 24 & 9 & 5 & 3 & \\
\cline { 2 - 8 } & Post & 30 & 9 & 5 & 6 & & \\
\hline \multirow{2}{*}{ LG - central } & Pre & 34 & 10 & 3 & 1 & & 2 \\
\cline { 2 - 8 } & Post & 40 & 4 & 2 & 4 & & \\
\hline \multirow{2}{*}{$\begin{array}{l}\text { LG - tempo- } \\
\text { ral }\end{array}$} & Pre & 14 & 26 & 4 & 3 & 3 & \\
\cline { 2 - 8 } & Post & 28 & 14 & 2 & 3 & 3 & \\
\hline
\end{tabular}

Table 1: Summary of grading results pre and post treatment. Abbreviations: MGD: Meibomian Gland Disfunction; LG: Lissamine Green. 
The MGD grade was statistically significantly lower after LLLT treatment (Wilcoxon matched pairs test, p < 0.001). Only $12 \%$ of eyes (6/50) had an MGD grade of 1 or lower before treatment; this increased to $50 \%$ (25/50) after treatment. This change was a statistically significant difference (Chi-squared test, $\mathrm{p}<0.001$ ). An MGD grade reduction of 1 or more was observed in $72 \%$ of eyes $(36 / 50)$, while $32 \%$ of eyes $(16 / 50)$ showed a decrease in grade of 2 or more.

LG grading was evaluated by location. The LG grade was statistically significantly lower after treatment in the nasal $(\mathrm{p}<0.001)$ and temporal $(p=0.02)$ locations, but not statistically significantly different centrally $(p=0.08)$. As can be seen in table 1 , the central location had the lowest LG grading before treatment.

Table 2 shows a summary of the findings related to the classification of the patients/eyes before treatment, so that relative changes in their classification could be better evaluated. For instance, using a cutoff score of $\geq 33$ on the OSDI to indicate severe dry eye, 12 patients would have been categorized as severe before treatment. This dropped to 7 patients at the post-treatment visit, a statistically significant reduction. The mean reduction in OSDI was higher in this subset of patients relative to the full cohort. Similarly, the changes in classification of dry eye based on TBUT $(\leq 6$ seconds for dry eye) and MGD grade (2 or higher for MGD dysfunction) could be analyzed for those eyes with a dry eye or MGD designation before treatment. A statistically significantly lower number of eyes were classified as dry based on TBUT and MGD grade after treatment. Note that the totals for the table cannot be summed, as patients may have had more than one criterion that met the classification for dry eye/MGD.

\begin{tabular}{|l|c|c|c|c|c|}
\hline $\begin{array}{l}\text { Classifica- } \\
\text { tion }\end{array}$ & Total & $\begin{array}{c}\mathbf{n} \\
\text { Pre }\end{array}$ & $\begin{array}{c}\mathbf{n} \\
\text { Post }\end{array}$ & $\mathbf{p}$ & Mean change \\
\hline OSDI > 32 & 25 & 12 & 7 & 0.01 & $\begin{array}{c}-11.8 \pm 14.6 \\
(-40 \text { to 13) }\end{array}$ \\
\hline $\begin{array}{l}\text { TBUT } \leq 6 \mathrm{~s} \\
\text { (one or both } \\
\text { eyes) }\end{array}$ & 50 & 23 & 7 & $<0.001$ & $\begin{array}{l}3.3 \pm 2.3 \\
(1 \text { to } 10)\end{array}$ \\
\hline $\begin{array}{l}\text { MGD Score } \geq \\
\text { 2 (one or both } \\
\text { eyes) }\end{array}$ & 50 & 44 & 25 & $<0.001$ & $\begin{array}{c}-1.2 \pm 0.9 \\
(-3 \text { to } 1)\end{array}$ \\
\hline
\end{tabular}

Table 2: Change in dry eye evaluation scores by pre-treatment classification.

Abbreviations: OSDI: Ocular Surface Disease Index; TBUT: Tear Breakup Time; MGD: Meibomian Gland Dysfunction.
Of the 25 patients (50 eyes) treated, there was a single complaint of mild ocular irritation over the course of the study. In addition, one patient had no response to treatment. She had been a treatment failure with numerous other modalities. Her meibography showed few glands remaining per lid (10 - 13) and these were atrophied to about $25 \%$ of normal size. She also showed concomitant grade 4 lid wiper epitheliopathy.

\section{Discussion}

The study described above was conducted to determine if LLLT could have any beneficial effect on meibomian gland function. LLLT has been studied in the past by the authors in a previous large case series, but it was administered in combination with Intense Pulsed Light (IPL) therapy, and a single treatment was provided to the majority of patients. The IPL used in that study did not require use of a gel, as most IPL systems do, due to the energies involved [18].

The advantages of using LLLT in the absence of IPL are several. Perhaps most importantly, IPL treatment must be managed with consideration of the level of pigment in the patient's skin, as hypopigmentation is possible in darker-skinned individuals [20,21]. There is no such risk with LLLT. In addition, the application of IPL is generally made below the lower eyelid, to reduce the risk of light penetration through the lid with subsequent absorption by ocular tissues. While rare, IPL can cause ocular inflammation [22,23] and without the proper safety precautions can cause damage to ocular structures that absorb light, such as the iris [24] and the retina [25]. With regard to efficacy, a recent Cochrane review of the effects of IPL suggests results in early studies may have overstated the potential benefits [26]. Finally, the application of traditional IPL generally involves the use of gels and eye covers, which can be inconvenient for both patients and staff. LLLT does not require these for safe use.

Results here showed a generally beneficial effect of LLLT after 3 consecutive 15-minute treatments. Most patients showed improvement in symptoms, and MGD/LG grading. Not all treatments were successful - in patients with a severe deficiency of meibomian glands, stimulation of the remaining glands through LLLT is likely to be only nominally effective.

The effectiveness of LLLT demonstrated here is consistent with the findings of Pult [16]. He not only established the effectiveness of LLLT treatment for dry eye, but also demonstrated the necessary thermographic evidence for such improvement. The results of LLLT 
seen here are also consistent with the expected anti-inflammatory effect of near infrared light in general. The anti-inflammatory and healing effects of LLLT have been established in several other medical specialties, notably dermatology and dentistry [27-29].

There are limitations to the study conducted here, sample size being one of them; only 25 patients were included in the chart review. In addition, because this was a review of patients treated in the clinic, adjunct pharmaceutical therapy was included. A prospective study of the response to LLLT alone or a direct contralateral eye study comparing LLLT to IPL would be of interest and is under consideration.

Follow-up time was also limited (5 weeks maximum). The effect of any maintenance treatment after the initial 3 sessions was also not investigated.

In summary, the use of LLLT in patients recalcitrant to previous treatments for MGD improved subjective symptoms and objective signs in the majority of those treated.

\section{Conclusion}

A short series of LLLT treatments over the course of a week produced significant improvements in tear breakup time and lissamine green/MGD grading scores measured three to five weeks after treatment. This was accompanied by improvement in the patients' subjective OSDI scores.

\section{Acknowledgements}

This study was supported with an investigator-initiated study grant from Espansione Marketing. Physicians Protocol (KGS) provided funding to Science in Vision to assist with data analysis and preparation of this manuscript.

\section{Financial Disclosures}

Dr. Karl Stonecipher is a consultant to Espansione Marketing S.p.A., Alcon, Allergan, Bausch Health Eyevance, Johnson and Johnson and Novartis. Dr. Richard Potvin is a consultant to Alcon and Carl Zeiss Meditec. Dr. Casey Komm has no relevant financial interests.

\section{Bibliography}

1. Craig JP., et al. "TFOS DEWS II Report Executive Summary". The Ocular Surface 15.4 (2017): 802-812.

2. Nichols KK., et al. "The international workshop on meibomian gland dysfunction: executive summary". Investigative Ophthalmology and Visual Science 52.4 (2011): 1922-1929.
3. Baudouin C., et al. "Revisiting the vicious circle of dry eye disease: a focus on the pathophysiology of meibomian gland dysfunction". British Journal of Ophthalmology 100.3 (2016): 300-306.

4. Mathers WD. "Ocular evaporation in meibomian gland dysfunction and dry eye". Ophthalmology 100.3 (1993): 347-351.

5. Nichols JJ., et al. "TFOS DEWS II Definition and Classification Report”. The Ocular Surface 15.3 (2017): 276-283.

6. Rong B., et al. "Long-Term Effects of Intense Pulsed Light Combined with Meibomian Gland Expression in the Treatment of Meibomian Gland Dysfunction". Photobiomodulation, Photomedicine, and Laser Surgery 36.10 (2018): 562-567.

7. Mandal P., et al. "Drugs - Do we need them? Applications of non-pharmaceutical therapy in anterior eye disease: A review". Eye 41.6 (2018): 547.

8. Tauber J., et al. "Comparison of the iLUX and the LipiFlow for the Treatment of Meibomian Gland Dysfunction and Symptoms: A Randomized Clinical Trial". Clinical Ophthalmology 14 (2020): 405-418.

9. Kim WS and Calderhead RG. "Is light-emitting diode phototherapy (LED-LLLT) really effective?" Laser Therapy 20.3 (2011): 205-215.

10. Farivar S., et al. "Biological effects of low-level laser therapy". Journal of Lasers in Medical Sciences 5 (2014): 58-62.

11. Ablon G. "Phototherapy with Light Emitting Diodes: Treating a Broad Range of Medical and Aesthetic Conditions in Dermatology". The Journal of Clinical and Aesthetic Dermatology 11.2 (2018): 21-27.

12. Huang A., et al. "Light Emitting Diode Phototherapy for Skin Aging". Journal of Drugs in Dermatology 19.4 (2020): 359-364.

13. Sun Q., et al. "Red light-emitting diode irradiation regulates oxidative stress and inflammation through SPHK1/NF- $\mathrm{kB}$ activation in human keratinocytes". The Journal of Photochemistry and Photobiology B: Biology 186 (2018): 31-40.

14. Toyos R., et al. "The Effects of Red Light Technology on Dry Eye Disease Due to Meibomian Gland Dysfunction". JOJ Ophthalmology 3.5 (2017): 555624.

15. Stonecipher K and Potvin R. "Low level light therapy for the treatment of recalcitrant chalazia: a sample case summary". Clinical Ophthalmology 13 (2019): 1727-1733. 
16. Pult H. "Low-Level Light Therapy in the Treatment of Meibomian Gland Dysfunction". Investigative Ophthalmology and Visual Science 61.7 (2020): 99.

17. Schiffman RM., et al. "Reliability and validity of the Ocular Surface Disease Index". Archives of Ophthalmology 118.5 (2000): 615-621.

18. Stonecipher K., et al. "Combined low level light therapy and intense pulsed light therapy for the treatment of meibomian gland dysfunction". Clinical Ophthalmology 13 (2019): 993999.

19. Bron AJ., et al. "Grading of corneal and conjunctival staining in the context of other dry eye tests". Cornea 22.7 (2003): 640650 .

20. Thaysen-Petersen D., et al. "Side effects from intense pulsed light: Importance of skin pigmentation, fluence level and ultraviolet radiation-A randomized controlled trial". Lasers in Surgery and Medicine 49.1 (2017): 88-96.

21. Alam M. "What devices to use or not use in skin of color". Seminars in Cutaneous Medicine and Surgery 35.4 (2016): 218-222.

22. Crabb M., et al. "Intense pulsed light therapy (IPL) induced iritis following treatment for a medial canthal capillary malformation". Australasian Journal of Dermatology 55.4 (2014): 289-291.

23. Pang AL and Wells K. "Bilateral anterior uveitis after intense pulsed light therapy for pigmented eyelid lesions". Dermatologic Surgery 34.9 (2008): 1276-1279.

24. Jewsbury $\mathrm{H}$ and Morgan F. "Uveitis and iris photoablation secondary to intense pulsed light therapy". The Canadian Journal of Ophthalmology 47.4 (2012): e13-e14.

25. Maganti N., et al. "Macular Hole Formation Associated With Intense Pulsed Light Therapy (2019).

26. Cote S., et al. "Intense pulsed light (IPL) Therapy for the treatment of Meibomian gland dysfuction". Cochrane Systematic Review-Intervention (2020).

27. Walski T., et al. "The effect of Red-to-Near-Infrared (R/NIR) irradiation on inflammatory processes". International Journal of Radiation Biology 6 (2019): 1-37.

28. Dompe C., et al. "Photobiomodulation-Underlying Mechanism and Clinical Applications". Journal of Clinical Medicine 9.6 (2020): E1724.
29. Avci P., et al. "Low-level laser (light) therapy (LLLT) in skin: stimulating, healing, restoring". Seminars in Cutaneous Medicine and Surgery 32.1 (2013): 41-52.

\section{Assets from publication with us}

- Prompt Acknowledgement after receiving the article

- Thorough Double blinded peer review

- Rapid Publication

- Issue of Publication Certificate

- High visibility of your Published work

Website: $\underline{w w w . a c t a s c i e n t i f i c . c o m / ~}$

Submit Article: www.actascientific.com/submission.php

Email us: editor@actascientific.com

Contact us: +919182824667 